\title{
SUBJEGT INDEX
}

agriculture $254,344,449,499,504,508-$ 509

Alsace-Lorraine 36-37, 52, I2I, I23, I73, I88, 27In, 359, 382, 469n, 47 In, 477, 524

Altona I57, 299, 369n

America (see United States of America)

anarchism/nihilism $164-165,326,337$, 346, 368, 371, 379

Anglophobia/anti-British sentiments (see also Germany and Great Britain) I6I7, 52-55, II7-II8, I67, 24I, 244-245, 263,3 Io, 315, 324-325, 334, 363-364, $367,375,377^{-380}, 388,5^{1}{ }^{1-} 5^{12}$

anti-Semitism (see Jews)

Anti-Socialist Laws and measures I4, I38I 4 I, 246-248, 25on, 326-328, 336-339, $367-368,37 \mathrm{I}^{-} 37^{2}, 39^{-}-39 \mathrm{I}, 4 \mathrm{I} 4,49^{8}-$ 500

Arab-Tabia ${ }_{15} 6$

Armenia $35^{8}$

army and military affairs (see also Austro-

Prussian War; Franco-Prussian War): of Baden 236; of Bavaria 236-237, 264, 457-458, 494-495; of France 54, 74-76, 99, IOI-I03, г22-I25, I72, I87, I90, 236-237; of German Empire (see also German Empire, military laws) 6o-6I, 63-64, 75-77, 87, I03-IO4, I23I25, I40, I76-I77, I82-I83, 219, 24I, $243,254,29 \mathrm{I}-292,42 \mathrm{I}, 424-425,507-$ 508; of Great Britain 34, 54-55, I24I25, I56, I78, I83-184, 262-263, 313, $33^{\mathrm{I}}, 364,366,383,4 \mathrm{I} 9,5^{\mathrm{II}-} 5^{\mathrm{I} 2}$; of Hesse 206, 225, 236, 238-239, 264-265; of Prussia 54-55, 234, 29I; of Russia (see also Russo-Turkish War) 125, I28, 3I5; of Saxony 269, 29I-292, 312-3I3; of Württemberg 236, 395, 422-423

Arolsen 422

assassinations (attempts) I4, 94-95, 98, I38, I4I, I53n, I64, 247-249, 336-337, 368n, $4 \mathrm{I} 4$

Australia 129, 303n
Austria-Hungary (see also army and military affairs; Austro-Prussian War; Bohemia; Hungary; League of the Three Emperors): and France 93-94, IOI; and German Empire (see also Dual Alliance) I8-I9, 33-34, 54-55, $63,68,70-71,93-94,99^{-100}$, I04, Iо8, III-II4, I30, I36, I49-I50, I59-I62, I69-170, 244, 316-317, 365-366, 388, 435, 472-473; and Great Britain 78, I2O-I2I, I35, 366; and Italy IOI-IO2, 359; and Russia 70-7I, III-II2, I2O, I23, I32, I36-I37, I47-I48, I5I, I60I63, I94-195, 244, 316, 359, 472-473; emperor (see name index: Franz Joseph I); government $33^{-34}, 55 \mathrm{n}, 93^{-94}$, II2, I59

Austro-Prussian War 37n, 57, 6o, 77, 87, 93, 98, 149, 195-196, 205n, 222, 243, 256, 3I $4,43 \mathrm{I}$

\section{Baden (Austria) I48, I50}

Baden (see also army and military affairs): and German Empire (see also German Empire: November Treaties; reserved rights) II -II $^{-1}, 2 \mathrm{I} 5,225,257,26 \mathrm{o}, 348$, 405; and Prussia 222, 225-226, 256; chambers 228, 258-259, 265-266; grand duke (see name index: Friedrich I); ministry 200, 265; religion and religious policy 20I, 208-210, 212, 228

Baden-Baden 58, 83n

Balkan crisis (see also Russo-Turkish War) I08, III-II2, II3n, II6, II7n, II9-I2I, $35^{\circ}$

Baltic 6I

Bamberg 435

banks/stock exchange 35,4 I, 92-93, IIon, II $5,226,34^{\mathrm{O}}, 347,35^{\mathrm{I}}, 353^{-3} 34,44^{8-}$ $45^{2}$

Bautzen 29I

Bavaria (see also army and military affairs): and Austria I9, 316,435, 494-496; 
and France I89-I9o, 469-47I, 5095IO, 5I8, 524; and German Empire (see also German Empire: November Treaties; reserved rights) II, 22, 59, 85, II4-II5，I57，2I5，333，396-397，399， $407,429-43^{\mathrm{I}}, 43^{6}-437,453-454,457^{-}$ $470,484-487,492,494-496,502-504$, 508-509, 5I3-5I7, 52I, 525-528; and Prussia II, 39-40, 465, 475, 484-486, 494, 5I4-5I6, 526; chambers IO9-IIO, $396,429-43 \mathrm{I}, 437,453,45^{8}, 460-$ $462,464,466,482-487,49 \mathrm{I}-494,502-$ 503, 506-508, 514; Concordat 208, 455, 489-49o; constitution 432-433, 46o-46r, 466, 483-484, 489-49o, 528; diplomatic relations II, 22, 39-40, 298, 447, 452-454, 468-470, 50I-503, 505, 509-5II, 5I3, 517; king (see name index: Ludwig II); ministry $453^{-456}$, $465,470-47 \mathrm{I}, 482-485,487,494,5 \mathrm{OI}$, 506-507, 526; regency 527; religion and religious policy 208, 432-439, $44^{8-452,455^{-457}, 476,484,486,488-}$ $49^{\mathrm{I}}, 495,5^{\mathrm{O} 6}-5^{\mathrm{O}} 7,5^{\mathrm{I}} 3,5^{\mathrm{I}} 5,5^{2 \mathrm{I}}$

Belfort 55, 2 I8

Belgium 37, 95, 98-I0о, I30, 4Io, 480-48I

Berg 465, 5 OI

Berlin 7, I0, I2, I7, 20-22, 32, 33n, 39, 4I,

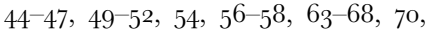
$72,77,80-83 \mathrm{n}, 85,88,9 \mathrm{I}, 96-97$, го5, IO9, II2, II4, II6, I20, I22-I27, I3O, I 35 , I40-I 43 , I46, I49-I50, I52, I59, I70, I72, I74-I75, I77, I79-I81, I83I84, I88-I9o, I94, I96, 200-20I, 203204, 207, 226, 233, 235-237, 240, 246, 250, 252, 254, 273, 285, 287, 290-294, 298, 309, 319, 322, 324, 327-330, 333$335,337,347,349-353,366-368$, 37I, 387, 405-407, 409, 4I2, 4I4, 4I7-4I8, $424-425,433,435-436,44 \mathrm{I}, 444,447$, $453,466-467,470-47 \mathrm{I}, 476,484,487$, $509,5 \mathrm{I} 3,522-523$; British embassy $2-$ 4, 6-IO, 20-2I, 23, 34-36, 44-47, 8283 , I33, 492; Congress/Treaty of I6, 20, I34-I37, I47, I50, I55-I57, I62-I63, I72, 246n, 25I, 33In, 334, 358, 364n

Berne 296, 502n

Bessarabia 33I

Bingen 254-255

Black Sea I2 I, I34, I36, I63, I95

Blue Books I22-I23

Bohemia I49, I70, 239, 435

Bologna 445
Bosnia II2n, I36, I47n, I62, 3IIn, 3I7n

Brazil 522

Breslau (Wrocław) 96, 29o, $35^{2}$

Brunswick 27I; succession question $5 \mathrm{I}$, I42

Brussels 39, 66, 8o, 98-99, I97, 478n, 480

Bulgaria 2I, II6n, I34n, I36, I47n, I56n, 239-240, 25I, $3^{\mathrm{I} 7 \mathrm{n}}, 35^{8 \mathrm{n}}$

Calais 29I

Canada 22, 6I-62

Canossa (Bismarck's speech) I86, 284, 36o, $362,376,52 \mathrm{I}$

Canton 370

Carlsbad, conferences and decrees 28I, 304

Cassel I8I, 200

Catholic Church (see Roman Catholics)

censorship (see press)

Charlottenburg I40

Chemnitz 274-276, 288, 304

Cherbourg I59, I73

China I29

civil code/civil law 252-253, 277-28o, 396, $459-462,466-467$

clergy $43,48,56,69,73,79^{-8}$ I, 89, 95, гоо, I54, I56, 202, 2IO, 2I $, 226,229,28 \mathrm{I}-$ 282, 295, 305, 362, 376, 4II, 4I5-4I6, $43^{2}-433,43^{6}-437,44^{2}, 455^{-456,476,}$ 488-49I, 494, 498-499

Coblence (Koblenz) 58, 254-255

Coburg 5-7

Cologne 29-30, 79, I53, 2II, 290, 363, 5I9 colonies/colonial ambitions 22-23, 85-86, I05, IIо-III, I28-I29, I60, I67, I78, I84-I85, 307, 357, 373, 512

commerce (see trade and commerce)

conservatives (see also parties, Freikonservative Partei) 42, 47-48, 56-58, 7I, 8I, 87-89, IOI, I38-I39, I43-I44, I68-I69, I85I86, 20I-202, 238, 256, 258-259, 265266, 285, 292, 309-310, 315, 3І7, 329, $33^{\circ}, 33^{8}, 35^{\mathrm{I}}, 359,3^{62}, 37^{2}-374,37^{8-}$ $379,381,390,429,437$

Constance 204

Constantinople I8, 66, I20, I5I, I56-I57, I59, I63, I67, I76 n, 29I, 3I5, 3I7, 33 I

constitutionalism/constitutional conflicts I4-I5, 48, 53, 8I, I57n, I58n, I73I74, 244, 246-247, 279-28I, 284-285, 288-289, 319, 332-334, 338-339, 342, $345^{-}-346,387,49^{2}-494,463$

Copenhagen 355, 370

Crete ${ }^{6} 63$, I95n

currency/gold standard 2I4-2I5, 347 
Customs Union (Zollverein) II5, I58, 35I, $354,357,361,370$

customs, customs' laws, duties and tariffs (see also free trade; protectionism) $\mathrm{I}_{42}$ I44n, I49, I57-I58, I62, I72n, 225, 23On, 257, 26I, 32In, 325, 34I-342, 344, $349,369-370,373$

Danube 70, I2I, I27-I28, 3I5, 33I

Danubian Principalities I2I

Danzig (Gdańsk) I7I, 38I

Darmstadt (see also Hesse-Darmstadt) 2-3, 5-7, 9, I2, I7, 20-2I, I97-I98, 2I4, 237, 24I, 248, 25I, 253, 265, 296, 4I 6

Delagoa Bay 105

democrats (see also parties, Deutsche Volkspartei) 220, 237, 257-259, 27I, 304, 39I, 4I4, $42 \mathrm{O}, 423-424$

Denmark 52, I2I, I42, I46n, 34I

Diet of the German Confederation 3, 222, 406

diplomatic practice and protocol $4^{-8}, 3^{-}$ 3I, 34-36, 39-40, 44-46, 65-66, 82-83, 9I-92, I09, I22-I23, I52, I89, 273-274, 293-299, 3І I-3І $3,329,376-378,389$, 395, 402-403, 406-407, 416-4I7, 447, $477^{-480}, 5 \mathrm{OI}-5 \mathrm{O} 2,505,509-5 \mathrm{II}$

Dreikaiserbund (see League of the Three Emperors)

Dresden IO, I2, I7, 85, 269-270, 272, 28I, 285, 288, 290, 292, 294-299, 304, 319, $326-327,330-33$ I, 337, 340, 348, 350$35 \mathrm{I}, 353-355,36 \mathrm{o}, 37 \mathrm{I}-374,37^{6-378}$, $3^{8} \mathrm{I}-382,384,389,464$; British mission 2-7, 273-274, 294-299

Dual Alliance I9, I46-I52, I59, I6I-I63, I66, I70-I7I, I90, 349, 359, 42I, $5^{\mathrm{I}} 3^{\mathrm{n}}$

Eastern Question I6, 20-2I, 55, 6I, 68, 707I, III-II2, II4, II6-II7, II9-I23, I26, I30, I32-I37, I5I, I55-I57, I59, I6II63, I66-I68, 306, 315-317, 320-321, $33^{\mathrm{I}-} 33^{2}, 35^{\circ}, 35^{8-}-359,363-364,47^{-}-$ 473,524

Eastern Rumelia I47n, I56-I57

Egypt (see also Suez Canal) I6, 22-23, IIoIII， I2I， I35，I55，I66-I68, I83, I75$\mathrm{I} 78,263,306-307,383,5^{\mathrm{II}-5}{ }^{\mathrm{I} 2}$

Eisenach 300-30 I

Elbe, navigation act $157,369 n$

Elberfeld 299 elections (see also franchise) 8, I4, 38, 4I, I38-I39, I43, I68-I69, I74, I98, 203, 206, 213, 220, 237-238, 257-259, 265266, 28I, 322, 325-326, 337, 352, 372$374,38 \mathrm{I}, 4 \mathrm{I} 5,420,423-424,450,476$, $482-484,49 \mathrm{I}, 493,499,506,5 \mathrm{I} 7$

Ellwangen $4 \mathrm{II}$ emigration 22, 6I-62, 97-98, I6o, 366

Ems 33, 67, 83n, I07, I93-194; Dispatch $33 \mathrm{n}$

England (see Great Britain)

Erbach 253

Erzurum I28

Esslingen $4 \mathrm{I} 5$

exhibitions 67n, 70, I2I-I22, I35, 335, 50 I

Falk Laws (see May Laws)

Faulquemont 264

Federal Council (Bundesrat) 9, 47, 59, 79n, 8I, 88, I40, I43n, I57-I58, 246, 25926I, 279, 285, 290, 3I4, 333-334, 339, $34 \mathrm{I}, 344,348,357,367,369,372,396-$ 400, 406-407, 409, 4I4, 418-4I9, 436, 458-462, 466-468, 487, 508; blocking minority $9,348,396-400,407,45^{8-}$ 459,487

Filehne 69

fortifications/fortresses $35,37,177, \mathbf{1} 87 \mathrm{n}$, $239,284,287,342$

France (see also army and military affairs): and Germany (see also Alsace-Lorraine; Franco-Prussian War; 'War-in-Sight' crisis) I6-I8, 73-76, I2 I-I23, I30, I35, I5I-I53, I6I, I63, I66-I68, I72-I73, I75, I86-I90, 218, 245, 250, 323, 384$385,388,433,467,469-472,474^{-}$ $475,501,518,523-524$; and Great Britain I7, 34-37, 53, 55, 6о-6r, 63, 75, 77-78, 82, 84, Іо3-І04, Іо6, Іі6, I5I， I55-I56， I66-I68， I72， I76， I78, 347; and Russia 84, I22, I35, I53, I59, I6I, I63, I7I, I94-195, 385, 42I, 507; government/domestic affairs $73^{-}$ 75, 93, го0, 135, 179, 189, 194, 346$347,382,384-385,43^{8}, 44^{6-} 447,470^{-}$ 47I, 518, 522-523; Napoleonic Wars 33,76 , I8o; war of revenge $17,54^{-56}$, $60-6$ I, 63, 74, 80, 99, Iог, II3, I2 I, I59, I73, I87-I88, 217, 221, 243-244, 385, $44^{2}, 504,507,5^{18}$

franchise/electoral laws 4I, 259, 30I, 373, $423,482-484,487,49 \mathrm{I}, 493$ 
Franco-Prussian War (see also France, war of revenge; Frankfurt, Treaty of) I, 8-9, I6-I7, I9, 29-36, 53, 55, 57, 6o-6r, 73n, $87,93,95,98-99$, IO4, II7, I52, I80I8I, I89, I93-I97, 201, 2I8n, 223, 243, 264-265, 269-27I, 3IO, 312, 382, 385, 400, 42I, 43I, 457-458, 507, 514, 520; POWs 34-36, 272; war indemnity I7, 3I, 50, 54, 67, 77, 229, 276-277, 308, 32 I, $34 \mathrm{I}-342$

Frankfurt am Main 3, 58, 205-206, 237, 250, 254-255, 440, 5I5; Treaty of I, 9,

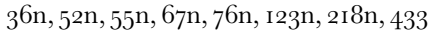

free trade $344-345,354,370$

Freiburg im Breisgau 201, 210, 238

Friedrichsruh 77n, I59n, 344

Fulda $43,69,282-283$

Gaesdonck 295

Garmisch 462-464

Gastein 51, 66-67, I48, I69-170

Geneva I78, 295n, 443

German Catholics (Deutschkatholiken) 43

German Confederation 3, 256, 337n, $45^{8}$

German Empire (see also Reichstag; Federal Council; civil code): budget and finances (see also Franco-Prussian War, war indemnity) 26I, 277, 285, 303-304, 308, 34I-345, 381, 386387, 420, 503; chancellor (see name index: Bismarck); civil and criminal procedure $25^{2-253}, 3^{\mathrm{I} 8}-3^{20}, 330$, 409, 45I, 459; constitution 9, I4-I5, $38,88 \mathrm{n}, 229^{-2} 3 \mathrm{o}, 235 \mathrm{n}, 26 \mathrm{o}^{-26}$ I, $279^{-}$ 280, 319, 322, 330, 332-333, 342, $348,365-366,369-370,387,396-401$, $405-407,453,45^{8-462,466-469,487}$ $490,5 \mathrm{I} 6$; criminal/penal code $23^{\mathrm{I}}-$ $23^{2}, 25^{2-253}, 275,304-306,43^{6-437}$, 448,459 ; diplomatic representation 22, 39-40, 67-68, 86n, 95, I52-I53, I64, I7I, 306, 329, 468-469, 502503, 5I3, 517; emperor (see name index: Wilhelm I); foreign policy (see also individual foreign states; Dual Alliance; League of the Three Emperors) I4I5, 2I-23, 54-57, 59, 62-63, 78, 83-84, 86, 93-95, 99-Iог, Iо4, Iо9, II9-I20, I32-I33, I55, I65-I68, I72-I73, I87, I89-I90, 242-244, 3I5, 431-432, 467469; imperial government Io, 59, 68, 91, 96-97, I33-134, I38-I39, I44-I45, I52-I53, I82-I83, 2I $8,220,232-233$,
$323-324,330,332-334,343,36$ I, 408409, 4I8, 424-425; military laws I4, I7, 76, 80-82, 89-9o, Iо3, I52, I59, 285287, 315, 342, 398, 503-504; November Treaties of I870 8, 39-40, I97, 256, 396n, 398-40I, 429-43I, 46I, 466-468, $484 \mathrm{n}$; occupational accident insurance I3, 365-366, 373-374, 386, 39on; proclamation of German Emperor I, 8, 30-32, 44, I97, 270, 429-43I; reserved rights of Baden, Bavaria, and Württemberg 9, 398, 405, 484-485n, 492, 515-5I6; unity of/nation building (see also individual German states) I, 6, 8II, 33, 44, 47, 53-54, 56, 66, 76, 84-87, 94-95, IOо, IO2, IO4, IIO, II4-II5, II8, 2OI, 208, 2IO, 2I4-2I5, 223, 225, 229230, 236, 238-239, 248, 26I, 286-287, $360-36 \mathrm{I}, 370,3^{84}, 400-402,4 \mathrm{I} 8,43 \mathrm{I}$, $43^{8}, 45^{8}, 4^{6} 3^{-46} 4,4^{6} 7,494^{-} 495,5^{\mathrm{I}} 4^{-}$ $5 \mathrm{I} 5$

Gießen 257

Glashütte $335-336$

Glauchau-Meerane (electoral district) 4I, 273,287

Gotha, congress and programme 2I9, 299, 325-326

Gravelotte, Battle of 312, 474

Great Britain (see also Anglophobia; army and military affairs; Ireland): and Franco-Prussian War I6-I7, 29, 3132, 34-36, 45n, 53, 6i, II7, г8о, 263, 270, 273-274, 395; and Germany (Anglo-German relations; see also Hesse-Darmstadt; Prussia; Saxony) I-2, I5-20, 30, 32, 44-45, 60-6I, $68, \quad 77-78, \quad 82-84,86, \quad$ I04, Iо6IO8, IIO-III, II3-II4, II7-II8, I22I25, I37, I46, I55, I64, I67, I80I82, 2I6-2I7, 227, 24I, 244-246, 263, $270, \quad 276, \quad 302-303,306-307,309-$

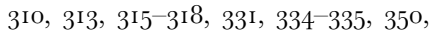
$357-360,3^{6} 3^{-} 364,3^{66-369,} 374-378$, $383,386,388,4 \mathrm{I} 9,480-48 \mathrm{I}, 5^{\mathrm{II}-}$ 512, 522-524; consular service 29-30, 34n, 48, 97n, I28-I29, I33; diplomatic representation and missions (see also Berlin; Dresden; Munich) I-8, I8, $2 \mathrm{I}^{-}$ 22, $34-36,44-46,82-83$, Iо9, I22I23, 273-274, 293-299, 376-378, 389, $395,477^{-480}, 5^{\mathrm{O} 5}, 5^{\mathrm{O} 9}-5^{\mathrm{II}}, 5^{22-523}$; government/domestic politics I3, I6, 78, 95, IO6, IIO-III, I37, 207, 247, 
309, 33I, 334, 357-36o, 363, 367-369, 375, 378-380, 386, 388, 390-39I, 48I; military attaché $4,34,54-55$, I24I25, I83-I84; subjects in Germany 29, I74-I75, 262, 294, 296-298, 477-478, $5 \mathrm{O} 2 \mathrm{n}$

Greece I56, I59, I62-I63, I67n, 239, $35^{8}$

Guelph fund/reptile fund 89, 28I, 289n, 327n, 334

Haiti 83

Hamburg I29, I57-I58, 342, 369-370, 37 I

Hanover (city) 3, 46

Hanover 3, 5I, 447; claims to sovereignty/ Guelph party I4I-I42, $44^{2}$

Heligoland 374-375

Herzegovina Io8, III-II2, II6, I36, I62

Hesse-Darmstadt (Grand Duchy; see also army and military affairs): and German Empire Io, I97-I98, 200, 2I5, 22I, 230-233, 235-236, 247, 25 I254, 260-26r; and Great Britain 5, 227, 262; and Prussia II, I96I98, 206, 223-226, 227, 239, 254255; and Russia 21, 222, 239-240, 25I; budget 226, 229-230; chambers 206, 224-227, 230, 235-236, 254; grand duke (see name index: Ludwig III; Ludwig IV); ministry Io, I96I98, 224, 23I-232, $255^{-256,} \quad 260-$ 26I; Oberhessen I97n, I99n, 256n257; Province of Starkenburg 257; religion and religious policy I98-I99, 202-204, 209-2IO, 2I6-2I7, 224-225, 232

Hohenschwangau 429, 496, 500, $5^{\text {IO- }} 5^{\text {I I }}$

Holland (see Netherlands)

Holstein 74, I46n

Holy See (Vatican; see also Roman Catholics) I2-I3, 38-40, 48-49, 55-56, 63, 73, 79, 95, IOO-I02, I2I, I45, I $53^{-\mathrm{I} 55}$, I68I69, I7I-I72, I85-I86, I90, 20I, 2I3, $4 \mathrm{II}, 4 \mathrm{I} 5,44 \mathrm{I}-447,45^{2-454}, 489,496-$ 498, 509-5I3, 519-52I; pope (see name

Honduras 82 index: Pius IX; Leo XIII)

Hubertusburg 287

Hungary I04, I I2, I36, $35^{2}$

Hyderabad I75

India III, I74-I75, 272-273

industry/factories I4, 20, IO2, 234, 238, $24 \mathrm{I}, \quad 247^{-248}, \quad 274^{-276}, \quad 3 \mathrm{OI}, \quad 335^{-}$
$33^{6}, 343^{-}-344,35^{\mathrm{I}}, 37 \mathrm{O}, 373,4 \mathrm{IO}$, 4I 4

International Workingmen's Association (First International) I3, 40-42, 48-49, $205,272-273,275,300-301,326$

Ioannina $\mathrm{I}_{3}$

Ireland/Irish I3, 37, I83-I84, 378-38o, 386,39 I, 522

Italy/Italians 55, 83, I00-IO2, I36, I5 I, I6 I, I66, 2 I3n, 359, 379, 446, 5II-5I3

Japan 83

Jesuits 80, I00-IOI, I98-I99, 20I-204, 295, $43^{8}, 443,446,49^{-}-49^{1}$

Jews and anti-Semitism 64, I58, 350-355, $372,374,403,448-449$

judicature and legal practice (see also German Empire, civil and criminal procedure) I4, 29, 9I-92, 96-97, 250254, 273, 287, 289, 292-294, 302-306, $3^{18-} 320,322-323,327-328,330,33^{6-}$ 339, 368-369, 451; Supreme German Court $322-323,330$

Kandahar 379

Karlsruhe 197, 210, 24I, 257

Katzenelnbogen 253

Kiel 85

Kissingen $66,476,496-497$

Königgrätz/Sadová, Battle of 314, 474

Königsberg 430

Königstein 287

Kulturkampf (see also May Laws) I2-I3, 37$3^{8}, 47^{-49}, 55,77^{-80}, 84,89,94^{-}$ $97,99^{- \text {IOI, }, ~} 444^{-\mathrm{I} 45}$, I53-I55, I68I69, I72, 20I-204, 207-208, 2I2-2I3, $220,224,228-229,234,282-284,294^{-}$ $295,35^{0}-35^{\mathrm{I}}, 357,362,376,390,408-$ 4I2, 438-447, 454-455, 457, 476, 482, $484,490,49^{6}-49^{8}, 5^{\mathrm{o}}, 5^{\mathrm{I}} 3,5^{\mathrm{I}} 9^{-}$ 52 I

Larissa 163

Lauenburg 77

Le Havre 53

League of the Three Emperors (Dreikaiserbund) $19-20,50-53,55,58,67 \mathrm{n}, 7 \mathrm{O}^{-}-7 \mathrm{I}$, 74, 93-94, II3-II4, II6, I36, I50, I6II63, I7I, I73, 243, 3II, 325

Leipzig II, 48n, I5n, 200, 237, 27I, 273n, 280, 299, 304, 31 1 -313, 3I5, 320, $328,330,332,336 \mathrm{n}, 348,354^{-355}$, 
37I-372, 474; university 280; state of siege $367-368,372$

liberals/liberalism (see also Manchester Liberalism; parties: Deutsche Fortschrittspartei; Liberale Vereinigung; Nationalliberale Partei) I4, 53, 63, 84, 87, I43, I64, I7I, I98n, 200-203, 207-209, 244, 246, 248-250, 263, 265, 309-3І $324,340,355,35^{8-}-359,373,37^{8-} 382$, 437, 443n, 448-45o, 486, 49o, 494

Livadia II9, I50-I5I, 25I

Loire 384

London 9, I3, I8, 39n-40, 45, 48-49, 6I, 65-66, 77, 82, 83n, 98n, 99n, Io6n, I25n, I34n, I43, I64, I72n, I75, 262, 272-273, 275, 294, 299, 306n, 317, 368n, $375^{\mathrm{n}}, 4^{6} 7 \mathrm{n}, 479^{-} 4^{80}, 5^{\mathrm{O} 2}, 5^{\mathrm{IO}}$

Lübeck 348

Ludwigsburg 422

Ludwigslust I59

Lusatia 380

Lützen 29I

Luxembourg $37,53,82$

Madrid 528

Magdala 3 I 3

Mainz (Mayence) I2, 36-37, 200, 202-204, 207, 21 2, 234, 237-239, 253, 257, 302

Manchester ${ }^{4} 6$

Manchester Liberalism 287-288, 3IO, 35 I, 355

Mannheim 204, 206, 260

Marienbad 39n, I48

Marienburg 52

May Laws (Falk Laws) I3, 69, 72-73, 798г, 89, 95-96, I44-I45, I53-I54, I69, I85-I86, 2 I3, 225, 284, 295, 360-362, $375^{-} 376,4 \mathrm{I} 6,49^{6}-49^{8}, 5^{\mathrm{I} 9}{ }^{-520}$

Mecklenburg-Schwerin 45

Mecklenburg-Strelitz 45

Metsovo 163

Metz 76

Mexico I84, 443

Mittenweida 288

monarchy/monarchical principle 2I, 63, I53n, I64, I73-I74, 220, 227, 278, 299n, $420,523,5^{27}$

Montenegro II7n, I34n, I56, I6In, 3II, $364 \mathrm{n}, 4 \mathrm{I} 7$

Munich I9, 25, 42, 95, I45n, I58, I97, 256, 299, 328-329, 335, 352, 397, 429, 432$434,447-45^{\mathrm{I}}, 453,45^{6}-457,464-465$, $469,476-478,493 \mathrm{n}, 495,499,5^{\mathrm{OI}}-5 \mathrm{O} 2$,
$5^{\mathrm{O} 5}, 5^{\mathrm{O}}{ }^{-5} 5^{\mathrm{I} 2}, 5^{\mathrm{I}} 4^{-5} 5^{\mathrm{I}} 5,5^{\mathrm{I}} 7,5^{26}, 5^{28}$; British mission 2-3, 6-9, II-I2, 477478, 509-5IO, 522-523; university I3, $43^{2}-433,45^{6}$

navy/German maritime power $23,55,85^{-}$ 86, I29, I40

Netherlands I2I, I30

Neva 359

New Zealand I29

newspapers (see press)

Nicaragua 82

Nikolsburg (Mikulov), preliminary peace of $25^{6}$

nobility/aristocracy $58 \mathrm{n}, \quad 76-77, \quad 87-89$, 294, 3I6, 39I, 424

North German Confederation 8, 35, 39, II5, I97, 222-223n, 256n, 277, 286n, $396 \mathrm{n}, 398 \mathrm{n}, 404 \mathrm{n}, 407 \mathrm{n}, 429,458 \mathrm{n}$, 46on, 468n

northern Germany 42, 8I, I99, 2IIn, 2I2, 254, 256, 263, 280, 300, 305, 330, 343, 378, 396-397, 399, 4IO, 4I5, 495

Nuremberg 352, 499

Offenbach 205, 237

Ohrid, Lake $35^{8}$

Old Catholic Church/movement I3, 4243, 7I-73, 89, 209-2I2, 224, 432-435, $455^{-457}, 4^{88 n}, 5^{\mathrm{I}} 5$

Olpe 69

Ottoman Empire (the Porte) (see also Balkan crisis; Bulgaria; Eastern Question; Egypt; Herzegovina; Montenegro; Russo-Turkish War; Serbia; Tunis) 20-2I, 6In, 70-7I, Io8, IIo-II4, II6, II7n, II9-I2I, I25-I28, I3I-I32, I34I37, I47n, I5I, I56-г57, I6I-I63, I67I68, I7In, I75-I76, 24I, 243, 25I, $3^{\mathrm{IO}-}$ $3^{\mathrm{II}}, 3^{\mathrm{I}} 7 \mathrm{n}, 3^{2 \mathrm{O}-} 3^{2 \mathrm{I}}, 3^{24} \mathrm{n}, 335^{\mathrm{n}}, 359$, $364,473,522$

Paderborn 69, 498

Palatinate (Pfalz) 222

Paris I4, 29, 33, 45, 67, 82, 86n, 95, 98, ro7n, IIO, II2, I52-I53, I65, I72, I80-I8I, I87, I88n, 2I6, 22 I, 23I, 256, 264, 27027I, 273, 298, 385n, 430, 44In, 467n, 469-47o, 472, 479, 50I, 502n, 507n, 5Ion; Congress and Treaty (I856) i 6 , I34, I36, I95n; La Commune 33, 37, 
23I, 247, 287n, 327, 346; World's Fair of 1878 I2 I-I22, I 35

parties (see also conservatives; democrats; socialists; liberals; ultramontanes): Allgemeiner Deutscher Arbeiterverein 2 Ign, 299, 302; Bayerische Patriotenpartei 437, 478n, 482-487, 490-494, 499, 5o6, 515-516; Christlich-Soziale Arbeiterpartei 322, 353, 374; Deutsche Fortschrittspartei 4I, I69, I86, 284-286, 288, 290, 294, 309-310, 32I, 327, 372-374, 433, 454; Deutsche Volkspartei 257, 420, 423-424; Freikonservative Partei 309-3IO, 3I5, 317, 329, 338; Katholische Volkspartei 499500; Liberale Vereinigung 256-258, 372, 378, 381-382; Nationalliberale Partei 42, $48,56,80-82,87-90,95,97$, го-IIo, I33-I34, I39, I42-I43, I69, I97, 2I3, 223-224, 230, 233, 235, 237-238, $247^{-248,256-259,265,285^{-286}, 288-}$ 294 , 300, 306, 309-312, 317, 32032 I, 324-325, 328-330, 333, 337, 342$344,347,35^{\mathrm{I}-35^{2}}, 35^{6}, 3^{60-} \mathrm{3}^{6 \mathrm{I}}, 37^{-}-$ $374,402,404,4 \mathrm{II}, 423,433,436$, 449, 454-455, 457, 459, 47o, 492-493, 5I5; Sozialdemokratische Arbeiterpartei 4I, I99-200, 2I9n, 275-276, 28I, 286288, 292, 294, 299-302; Sozialistische Arbeiterpartei Deutschlands 2I9-220, 23I, 237-238, 247-249, 299-302, 327, 336338, 372-374, 390-39I, 4I4-4I5, 499500; Zentrumspartei I2, 37-38, 43, 96n, I44-I45, I68-I69, I85-I86, I90, 219220, 237-238, 242, 257-259, 28I, 38In, $420,436,444^{-4} 45,470^{-} 47 \mathrm{I}, 49^{8}$

peasantry $25^{8}, 34^{2}, 3^{6} 7,44^{8}-45^{\circ}, 494$

Persia 63-66, 68

Pest I47n, 352, 473

Philippopolis (Plovdiv) I57

Poland/Poles/Polish party 47-48, 52, 76, $349,355^{-} 35^{6}, 3^{89 n}, 44^{\mathrm{I}}-44^{2}, 444$

police and prisons $4 \mathrm{I}, 48 \mathrm{n}, 49^{-5} \mathrm{O}, 58 \mathrm{n}, 69$, 73n, 79, 92, 96n, 97, I40, I84, 247n, 249-250, 253, 262, 264, 27I-273, 275, 28I, 284, 287-288, 300-30 I, 3I9, 327, 337-338, 368n, 39I, 403, 405, 4I4, 436437, 448, 45 I, 479, 499n

Posen (Prussian provincial state) 69, I99

Posen 44I, 498, $5^{\text {I9 }}$

postage/postal services 225, 398, 484n, $5^{\mathrm{I}} 5^{-5} 5^{\mathrm{I} 6}$

Poti I28

Potsdam 64, 65, I40
Prague I7o; Peace of (I866) 52n, I46, I49 press and journalism I7-I8, 23, 38, 42-43, $5^{\mathrm{I}-54}, 57,62,77,84,86,9^{\mathrm{I}-92}, 9^{6}$, IOIIO2, I05-IO7, II6-II8, I20, I28, I32, I4I, I44, I47-I48, I52, I59-I62, I70, I79, I87-I9O, 2OI-203, 2I7-2I8, 23I$232,243,245^{-246}, 288-29 \mathrm{I}, 3 \mathrm{O} 2,3^{\mathrm{IO}^{-}}$ $3^{\text {II }, ~ 317, ~ 319-320, ~ 332, ~ 334, ~ 35 ~ I, ~ 353-~}$ $354,3^{60}, 3^{6} 3^{-364}, 3^{88}, 4 \mathrm{II}, 45^{\mathrm{I}}, 469$, 480-48I, 486, 500, 5 ${ }^{\mathrm{II}-5}$ I $4,5^{2 \mathrm{I}}, 5^{2} 3^{-}$ 524; Imperial Press Law 8I, 280-282, 405-406; regulation of/censorship 8I, 89, I64, 234, 250, 280, 286, 30I, 3I9, 328, 368-369; semi-official/inspired press II7-II8, I28, I47-I48, I69, 264, 28I, 286, 288-290, 294, 309, 3I4, $33 \mathrm{O}, 334,344,35^{\mathrm{I}}, 37^{8-} 379, \quad 4 \mathrm{IO}$, 469n, 470, 474, 523; Allgemeine Zeitung 2I2, 242, 244, 294, 378, 455-456, 472-475; Berliner Börsen-Courier 353; Berliner Freie Presse 328; Berliner Tageblatt 264, 353; Constitutionelle Zeitung 28I; Correspondance de Berlin 36, 42, 94n, ıo8n, i26; Dagblad 355-356; Daily News 9I, 334-335, 366, 37I; Daily Telegraph 9In, 289; Darmstädter Zeitung 24I, 246; Das bayerische Vaterland 45I452, 499; Der Dresdner Volksbote 275, 28I, 294; Deutsche Allgemeine Zeitung 289; Deutsche Nachrichten 72; Deutsche Uhrmacherzeitung 336; Deutsche Wacht 354; Deutscher Reichsanzeiger und Königlich Preußischer Staatsanzeiger I05-I06, I08, I38; Dresdner Nachrichten 317, 344, 363, 382, 388; Dresdner Anzeiger 36o, 379, 388; Dresdner fournal 269, 335, 354, 364-365; Dresdner Presse 281, 294, 328; Edinburgh Review 307; Frankfurter fournal 379; Frankfurter Zeitung 250, 263, 319, Fränkische Tagespost 500; Freiheit I64; Germania 43, 96n, I54, I69, 2I3, 320; Hannoverscher Courier I54; Hessische Volksblätter 24I; Im neuen Reich 287, 355; Journal des débats 474; Kölnische Zeitung $3^{\mathrm{I}-32}, 5 \mathrm{I}, 53$, IOIn, I47, I55, I73, I84, I89, 2I6, 242, 244, 246, 3I5, 378, 474; La Correspondance de Genève 443-444; Le Mémorial diplomatique 279; Leipziger Tageblatt 289; Mainzer fournal 204, 2I3, 234; Mainzer Tageblatt 204; Mainzer Zeitung 242; Morning Post 509; Münchener Neueste Nachrichten 448-449; National Zeitung 42, I70, 232, 290, 
294, 3II, 334, 405; Nationalliberale Korrespondenz 289-290; Neue Frankfurter Presse 240-24I, 244, 246; Neue Freie Presse 279, 379; Neue Preußische Zeitung/Kreuzzeitung 3II, 4I9; New York Herald 9I; Norddeutsche Allgemeine Zeitung 5I, I47, I84, I86-I88, 264, 368, 388, 523-524; Pall Mall 9I; Pester Lloyd I47; Post IoIn, I47, I53-I54; Preußische Jahrbücher 97, 310, 330-33I, 354-355; Provinzial Correspondenz 8485, I25; Saturday Review 52; Schwäbischer Merkur 405; Spenersche Zeitung 5I; Staatsanzeiger für Württemberg 399; Süddeutsche Reichspost 20I; The Standard I83; The Times I8, 56, 6г, 85, го7, гі6, г3о, I88, 205-206, 2I6-2I8, 264-265, 270, 289, 3І5-3І6, 357, 368, 382, 388, 472, 474, 480-48I, 524; Volksstaat 299; Weimarische Neueste Nachrichten I54; World 91

protectionism (see also customs) I42-I44n, I69, 257, 32I, 323-325, 34I, 344-345, $349,373^{-} 374$

Protestants/Lutherans 63, 84, I98-I99, 208-209, 224, 238, 283, 294-295, 302,

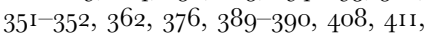
$439,446,449,507$

Prussia (see also army and military affairs; Franco-Prussian War; Austro-Prussian War; Posen; Rhine province; Silesia; Westphalia): and Austria 33-34, 435; and German Empire Io-I2, 44, 5759, 66, 76-77, 223, 43I, 445; and Great Britain 3o, 44; and Russia I93-I96, 350; chambers IO, 4I, 43, 47, 56-58, 88, I22, I48, I85-I86, 224-225, 342$343,35^{2}, 360,438,457$; constitution 38n, 43, 56-57, I73-174, 207n, 299, 30I, 304-305, 318, 342-343; king (see name index: Wilhelm I); ministry $3^{8}$, 43, 48, 59, 66, 68, I38, I73-I74, I82I83; Prussian supremacy 9, II, I96, 205-206, 210, 223-226, 229-230, 239, 244, 254-256, 280, 29o, 330, 355-356, 399, 402-403, 409, 478-479, 514-516; religion and religious policy (see also May Laws; Kulturkampf) 43, 72, 88, 207-2I0, 224

public opinion/popular feeling I5, I7-I8, 2I, 23, 5I, 53-54, 56, 6o, 7I, 80-8I, 9697, 99, IOI-IO2, I06, I22, I26-I27, I29,
I3I, I36-I37, I4I, I44, I48-I49, I7I, I73, I79, 2OI-202, 2I3, 24I-243, 247, 249, 279, 29I-295, 298, 3IO-3II, $3^{\mathrm{I} 5}$, $3^{20-} 3^{2 \mathrm{I}}, 334,337,343^{-344}, 35^{8}, 3^{60}$, $376,378,3^{81}$, 4I3, 4I8, 42I, 434, 472$475,480-48 \mathrm{I}, 503,5^{\mathrm{I} 2}-5 \mathrm{I} 3,5^{2 \mathrm{I}}$

Puerto Rico 86

railways $36,54 \mathrm{n}, 97 \mathrm{n}$, I05 $^{\mathrm{n}}, 34^{2}, 398,463$, $484 \mathrm{n}, 507$, 513n; plan for imperial railways IO, II4-II5, 233， 235-237, 254-255, 26I, 308-309, 325, 348-349, $373-374,404-405,412-4 \mathrm{I} 4,485-487$, 492-493

Red Cross I26

Reichstag 8-IO, I4, 36-38, 40, 43, 47, 66, 68, $76,80-82,89-90,94-97$, Іо3, Іо7, І23, I33-I34, I38-I39, I4I-I45, I48, I60, I65, I68-I69, I72, I82-I83, I86, I88, I96, I98-I99, 20I, 2I3, 220, 230-232, 236-238, 243, 257-26I, 27I, 277-278, 280, 284-289, 29I, 299, 303-304, 306, 3I7-3I8, 325-327, 330, 332, 34I-343, 345-346, 360, 369, 372-374, 379, 38I, 386-387, 398, 40I, 404, 4I4, 4I8-420, $433,436,44 \mathrm{I}, 444,457,459-462,467$, $470-47 \mathrm{I}, 483,49^{8}-500,5^{\mathrm{I} 2}$

religion and religious policy (see individual states; Kulturkampf; May Laws; Old Catholic Church; Protestants; Roman Catholics)

republicanism $63,87 n$, I39, I6o, 347, 379n, 420

Reuß 259n, 348

Reutlingen 304, 424n

revolutionary movements (see also anarchism; Paris Commune) 48-49, 87, 95, I27, I64, I78, I84, 238, 346-347, $35^{\circ}, 35^{6,} 5^{24}$

Rheims 55

Rhine: province (Prussia) 38, I54, I99, 255; river $37 \mathrm{n}, 222,252 \mathrm{n}, 255^{-255}$

riots $I_{3}, 49^{-5}$ o, I75n, 204-206, 238-239, 403

Roman Catholics/Roman Catholic Church (see also German Catholics, Kulturkampf; May Laws; parties, Zentrumspartei; Holy See; Jesuits; ultramontanes) I2-I3, 37-38, 42-43, 47-48, 84, I99, 202-204, 207-209, 2I $, 282-283,362-363,376$, 408, 4IO-4II, 4I5-4I6, 488-49I, 499, 520-52 I First Vatican Council/dogma 
of papal infallibility $\mathrm{I} 2-\mathrm{I} 3,43,72-$ 73 , 79, I99, 201, 210-2II, 282-283, $43^{2-435}, 43^{8}, 455^{-456}, 473$, 490; Verein der deutschen Katholiken 202-203, 2122I3

Romania I34n, I47n, I56, 239

Rome (see also Holy See) 38-39, I2I, I68, 2I3n, 362n, 434, 440-447, 453-454, $5^{\mathrm{IO}}, 5^{\mathrm{I} 2}-5^{\mathrm{I}} 3$

Rottenburg 4II, 4I5, 433

Russia (see also army and military affairs; Austria, and Russia; Eastern Question; France, and Russia; League of the Three Emperors; Russo-Turkish War): and German Empire (see also individual German states) I9-2 I, 32, 54-55, 6I, $63,70,83-84,99$, Io6-Io8, II3II4, II6-II9, I26-I28, I3I-I32, I36I37, I47-I52, I6I, I7I-I72, I78, I87I88, 24I-245, 28I, 310-3II, 3I5-3I $32 \mathrm{I}, 325,33^{\mathrm{I}-33^{2}}, 349^{-} 35^{\circ}, 35^{6}, 359$, 42I, 472-473, 507, 5I7-5I8; and Great Britain I8-20, 83-84, IO6-I08, IIIII4, II9-I2I, I3I-I33, I37, I63, 244246n, 317, 324-325, 350, 358; and Ottoman Empire I08, I20-I2I, I5I, I56, I59; emperor (see name index: Alexander II; Alexander III)

Russo-Turkish War (see also Balkan crisis; San Stefano, Treaty of) 2o, Io8n, II9n, I25-I28, I30-I35, I63n, 239-246, 33'

St Pauli I57, 36r, 369n

St Petersburg 44n, 63, 83n, 99n, IIo-III, I I9, I50-I52, I63, I64n, I78, I96n, 245, 4I6-4I7, 467n, 502

Samoa bill i6o, $357 \mathrm{n}$

Samsun I28

San Stefano, Treaty of I34-I37, I47n, 246, $35^{8}$

Saxony (see also army and military affairs): and Austria 329, 350, 359; and German Empire II, 85, II4-II5, 27027I, 277-28I, 284, 288-294, 298, 304305, 308-309, 31 I-3I4, 318-320, 322323, 329-330, 333-334, 339, 343-344, $348,3^{60-} 3^{6} \mathrm{I}, 3^{68-} 370,3^{84}, 3^{87}, 407$, 4I4, 459, 468, 486-487, 5I6; and Great Britain 309, 334, 376-378; and Poland 355-356; and Prussia II, I96, 269-27I, 280, 284, 286, 289-29I, 298, 322-323, 330, 355-356, 367-369; and Russia 2 I,
349-350; chambers Io, 276-280, 283284, 308-309, 330, 345, 357, 390-391; diplomatic representation $278,328-$ 329, 478n; king (see name index: Johann I; Albert); ministry 278-280, 289-29o, 314-3I5, 332; religion and religious policy $282-284,294^{-295}, 362,376$, 389-39o; Schleswig-Holstein 52n, 53, I46; Second Schleswig-Holstein War $57,60,87,3$ Io, 43 I

Schneeberg 287

schools and education (see also universities) Io, 47-48, I44, 208n, 2I 4, 2I 6-2 I 7, 224225, 228, 30I, 335-336, 340-34I, 353, 374, 380n, 382, 416, 439n, 488n, 493, $5^{06}-507,5^{1} 5$

Sedan, Battle of/Sedan day Io, 53, 6I, 8485 , I59, 2I4, 27I, 298, 382-383, 385, $474,5^{\mathrm{I} 7}$

Seine 359

Serbia II7n, I34n, I47n, I56, I62, 3II, 4I7, 5 I I

Silesia (Prussian provincial state) 38, 95, $3^{84}$

Slavs/Pan-Slavism 55, IO4, I2I, I27, 3II, $35^{8-}-359,507$

socialism/socialists (see also International Workingmen's Association; AntiSocialist Laws; parties) I3-I4, 4I, I39I4I， I99-202, 205-206, 219-220, 238, 247-249, 27I-276, 28I, 287-288, 303, $305,3^{\text {Io, } 320,} 3^{25}-327,33^{6-} 340,3^{65}$, 367-368, 386, 403, 4I4, 499

Sonthofen 463

Sorbs 380

South African Republic 23, I05, 379; First Boer War 366-367

southern Africa I6o, 366

southern Germany 38, 8I, I00, I97, I99, 207, 2I2, 2I5, 223, 229, 239, 277, 287, 297, 299-300, 359, 396, 398, 402, 405,

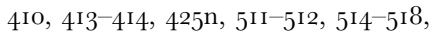
$520-52 \mathrm{I}, 524$

Spain 62-63, I90, 3I6n, 324n, 443, 522, 524

Städtetag I42-I43

Starnberg, Lake 462,464

strikes I3, 42, 200, 202, 274-276, 28I

Stuttgart 2-3, 6-7, 9, I2, I8, I97, 290, 299, $304,3 \mathrm{I} 7,359,395,402-403,407,4$ I5, 424

Suez Canal 22, i Io-III, 306-307, 309

Sweden I02, I2I, I24 
Switzerland I2I, 2I3, 295n, 30I, 324n, 336, 367

Syria I2I, I35

taxation and imposts I4, I33-I34, I43, I58, I82-I83, 225-226, 229-230, 257n, 277n, 301, 303-304, 342-345, 365, 373$374,398,404-405,414-415,4{ }^{18}-418$, $484 \mathrm{n}, 493,503-504,508$

Teheran 63,65

Tel el-Kebir, Battle of $178,263,383,5$ I I telegraph and telegrams $30^{-} 32,66$, Io6, IIо, II6, II9, I3In-I32, I35n, I55, I58, I68, I73, I83, I97, 216, 225, 246, 248249, 25I, 26I, 264, 289, 306, 325, 383, $39^{8}, 430,44^{2}, 453^{-} 454,476,5^{16}$

Tetschen 3 I7

theatres $3^{12-}-3 \mathrm{I} 3,502,526$

Thessaloniki (Salonica) I59

Thuringia 26I, 280, 300, 342

Tiber 359

Tiflis I28

Tirnova $25 \mathrm{I}$

tobacco monopoly I33n-I34, I75, 257n, 259-26I, 343-344, 373-374, 381, 508509,517

Tonga I29

Toronto 62

trade and commerce (see also banks; customs; industry; free trade; protectionism; currency) 50, 63-64, 86, 93, 98, го7, I22, I29, I49, I60, 200-20I, 205206, 2I4-2I5, 219, 225, 24I, 247 , 254-255, 26o-26I, 275, 287, 296-299, 30I-302, 3I IO, 32I-322, 335-336, 34I, 349, 35I, 353-355, 369-370, 4IO, 4I4, 459-46o, 499, 504, 508-509, 515-516; commercial treaties 63,68, I70, I72, 324,416 ; trade unions $200,288,340^{-}$ $34 \mathrm{I}$

Transvaal (see South African Republic)

Trebizond (Trabzon) 128

Trier 255

Trieste 7I

Tübingen 304, 408, 424

Tunis I30, I66-I68, I72

Turkey (see Ottoman Empire)

Tyrol 525, 527

Ulcinj (Dulcigno) 364

ultramontanes (see also parties: Zentrumspartei;

Bayerische Patriotenpartei) $\quad \mathrm{I} 3-\mathrm{I} 4,58,72-$

73, 77, 84, 94, 97-101, 106, I54, I97n,
I99, 202-204, 2II-2I $4,2 \mathrm{I}^{-2}-2 \mathrm{O}, 23 \mathrm{I}-$ 232, 234, 28In, 295, 35I, 376, 4II, 438$44 \mathrm{O}, 44^{2-}-443,447^{-} 44^{8}, 45 \mathrm{I}, 453-454$, $457,465,476,482-484,486,49^{\mathrm{I}}-494$, $499,5^{06}-507,5^{28}$

United States of America/Americans 5354, 56, 6I-62, 95, I83-I84, 293-294, $298,355,367,386 \mathrm{n}$

universities and professors 118, 198, 272, 295, 280, 310, 320, 330-331, 335, 354, $3^{81} \mathrm{I}, 408,424,43^{2-} 434,455^{-}-45^{6}, 473^{-}$ 475

Varzin (Warcino) 40, 5I, 56, 58n, 6o, 66, I02, IO7, I09, I33, I50-I5I, I77n, I94, 303, 333

Vatican (see Holy See)

Venice I02

Versailles (see also German Empire, November Treaties) 7, 29, 3on, 3in, $32 \mathrm{n}, 34 \mathrm{n}, 45,52$, I80-181, 197, 269270, 430, 445; Treaty of (I87I) 36n, 52, I97n

Vienna $33-34 \mathrm{n}, 67-68,70,76 \mathrm{n}$, 99n, IIо, I23, I46-I48, I50, I95, 246, 279, 317n, $328-329,335,349$ n, 352, 359, 397, 41 7 , $447,45^{8}, 467 n, 495,502$

'War-in-Sight' crisis I7-I8, 20, 98-I08, I50, I59, 2I7-2I9, 22I, 233, 4IO

Warsaw irgn, 356

Washington 62, 335

Weimar 359

Westphalia (Prussian provincial state) 38 , I99

Wiesbaden $38 \mathrm{I}$

Wittenberg 389

working and lower classes $13,40^{-}-42,4^{-}$ 50, I99-202, 204-206, 220, 237, 247n, $249,27 \mathrm{I}-276,278,288,301,325$, $34^{0}-34 \mathrm{I}, 365^{-366,} 373,390^{-}-39^{1}, 403$, 500

Worms 253, 257

Württemberg (see also army military affairs): and German Empire (see also German Empire: November Treaties; reserved rights;) 59, 397, 85, II4-II5, 215, 26o, 279, 348, 396-40I, 404-409, 412-4I4, $4^{16-} 420,422-423,459, \quad 468,486-$ 487, 515-516; and Prussia II, 402403; and Russia 416-4I7; chambers 396-399, 40I, 408, 415-4I7, 423-424; constitution 4I5, 423-424; diplomatic 
representation 397-398, 406-407, 416- Würzburg $45^{6}$ 4I7; king (see name index: Karl I); ministry 4I6-4I7, 423; religion and religious policy 408-4I2, 4I5-4I6, 434

Zollverein (see Customs Union)

Zwickau 27I, 287 